Volume 8, No. 7, July - August 2017

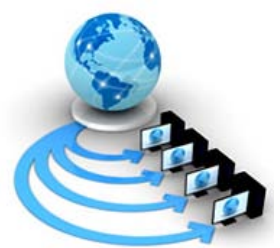

International Journal of Advanced Research in Computer Science

RESEARCH PAPER

Available Online at www.ijarcs.info

\title{
SURVEY ON CLUSTERING ENERGY EFFICIENT IN WIRELESS SENSOR NETWORKS
}

\author{
V.Saranya \\ Research Scholar, \\ Department of Computer Science, \\ Bharathiar University,Coimbatore-46.
}

\author{
P.B.Pankajavalli \\ Assistant Professor, \\ Department of Computer Science, \\ Bharathiar University,Coimbatore-46.
}

\begin{abstract}
A Wireless Sensor Network is a consisting of spatially distributed autonomous systems with a communication for monitoring and recording conditions at diverse locations. WSN is built of nodes from a few to several hundred or even thousand, where each node is connected to one sensors. WSN is an emerging and attractive research area in which several applications such as health care, smart home, agriculture, and military are making use of it. Energy efficiency is one of the most important issues in wireless sensor network. A cluster is a group of servers and other machines act like a single system. Clusters enable high availability, load balancing and parallel ${ }^{\text {processing }}$ in certain situation. Clustering algorithm is used to reduce the energy consumption and improves the lifetime of the network. Clustering reduces traffic flow, minimizes energy consumption, increase residual energy and extend the lifetime of the sensor network. This paper includes study of various energy efficient clustering algorithms in Wireless Sensor Networks.
\end{abstract}

Keywords: WSN, clustering, LEACH, TEEN

\section{INTRODUCTION}

Wireless Sensor Networks (WSN) has been applied in variety of applications, such as healthcare, smart home, agriculture, and military. A WSN is a network system of spatially distributed devices using wireless sensor node to collaboratively collect, process, and transmit physical or environmental conditions [1]. Sensor nodes are used in monitoring physical phenomena like temperature, humidity, vibrations and so on. Energy consumption is one of the biggest constraints of the wireless sensor nodes. Many researchers are working in energy efficient sensor nodes, development of energy efficient network protocol and topology. Power is consumed by a sensor node to sense processing and to transmit data. Data transmission is the most energy consuming operations. Introduction of clustering approach in the WSN data transmission will reduce the energy consumption. Clustering is one of the important methods for prolonging the network lifetime in WSNs. It involves grouping of sensor nodes into clusters and Cluster Head and communication will happen between $\mathrm{CH}$ and Base station (BS) [2]. Figure1. Shows an cluster head selection in WSN. CHs collect the data from respective cluster's nodes and forward the aggregated data to BS. A major challenge in WSN is to select appropriate $\mathrm{CH}$ [3].

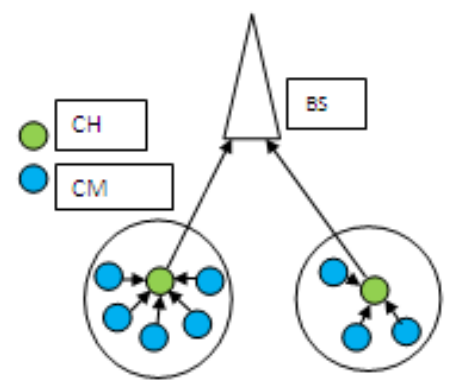

\section{Figure1. Wireless Sensor Networks}

\section{ENERGY MANAGEMENT}

The routing protocol uses some techniques to improve the energy-efficiency and network lifetime of a Wireless Sensor Nodes (WSNs). A few techniques of energy management are discussed below:

\section{Energy model}

Energy modeling is the computerized simulation of building or complex, this is focused on energy consumption [4]. The energy model produces a better estimation of remaining energy in each node. This is used to improve the network performance and node lifetime.

\section{Minimize the collision}

A collision is a network attempting to transmit data at exactly the same time. In routing protocol, the data should send between source and the destination without any interference. The protocol has to make sure that each node should communicate in the congestion-free environment.

Otherwise, it may lead to re-transmission of data, which directly affect the energy-efficiency of the network. So, the data sent should be free from congested path.

Minimize the control packet overhead

Signal transmission of the sensor node consumes the maximum amount of energy. The neighbor information, route discovery and maintenance in the routing protocol involve plenty of control packets exchanged between sensor nodes [3][5]. The routing protocol needs to restrict the unnecessary flow of control packet in the network. The size of the control packet may also be minimized.

\section{Multi-hop communication}

The direct data communication always consumes more energy than multi-hop communication in WSN. To communicate directly, the sensor node has to maximize the radio transmission power, which directly increases the 
energy consumption at each node. The routing protocol used to take care of these issues to improve energy-efficiency.

\section{Energy-aware MAC protocol}

A sensor network is capable of processing some information, gathering sensor information and communicating with other connected nodes in the network. The sensor node generates the data and forwards it to the sink. When the sensor nodes are not sensing or routing, they need to switch into sleep mode. The MAC protocol is required for the energy conservation in the network.

\section{Load balancing}

Energy model of each node is calculated using their residual energy. The routing protocol has to manage the load among the sensor nodes. More works can be assigned to an energy-rich node and workload can be reduced from the nodes having less residual energy. The load balancing technique has to be applied to improve the energyefficiency.

\section{Transmission range adjustment}

Multi-hop network in the WSN data should reach the destination through the intermediate nodes. Generally, that the next available relay nodes are always in close vicinity of the sensor node. Sending the data with maximum power, the transmission power can be readjusted using the Received Signal Strength Indicator (RSSI) [6]. It is used to measure of signal strength which can vary due to multipath, interference or other environmental effects; it may not give a true indication of communication performance or range. This technique can reduce the energy consumption and helps to improve the network lifetime.

\section{Data aggregation}

The data packets can be aggregated at some point and can send the aggregated data to the sink node. This technique of aggregating similar data decreases the traffic in the network. The reduced traffic decreases the collision and energy consumption. The routing protocol is used to implement the aggregation technique to prolong the lifetime of the network.

\section{DESIGN CHALLENGES IN CLUSTERING ALGORITHMS}

In the dynamic and energy constraint network, it is a challenging task to develop a routing protocol. A few issues and challenges for routing in WSN are discussed below:

\section{Energy constraint}

Sensor devices have limited battery-power. A large amount of energy is consumed during data transmission [7]. In route discovery and maintenance phase, a significant amount of energy is consumed. The lifetime of the network depends on the total energy consumption by each node. When node energy reaches below a certain level, it will become nonfunctional and affects the performance of the network. Therefore, it is a big challenge for routing packets in WSN.

\section{Bandwidth constraint}

WSN consists of a large number of sensor nodes, allocating a bandwidth for each link is a challenging task. In route discovery and maintenance, an enormous amount of control packets has to be broadcasted among the sensor nodes [7][8]. The bandwidth allocation process depends on the number of links and the amount of data they can communicate. This is one of the challenging tasks in routing packets.

\section{Limited Energy}

WSN have a limited amount of energy sources, it is very difficult to recharge or replace their batteries. This affects the efficient data transmission in WSNs. This can be overcome by balancing the energy consumption in sensor nodes by optimizing the cluster formation.

\section{Crowded center effect}

The data transmission from source to a sink in WSN is many-to-one relationship. In multi-hop WSN, each sensor node forwards the data to the sink through intermediate sensor nodes. The sensor nodes near the sink always relay on large number of data [9]. Therefore, they consume more energy than the remaining nodes and finally they die. This is one of the challenges in the WSN.

\section{Storage}

The sensors have limited amount of storage and communication is costly. WSNs require the data to be transferred from sensor nodes to a centralized base station. Sensor network has continued sensing and storage of data [10]. The storage is one of the challenges in WSN.

\section{Security}

WSN have many applications in military, homeland security and other areas. Most sensor networks actively monitor their surroundings, and it often deduces information other than the data monitored. Secure data transmission is one of the critical issues for WSN.

\section{ROUTING TECHNIQUES}

There are different ways to distinguish and classify the clustering algorithms used in WSN. Most of the known clustering algorithms for WSNs can be distinguished on the basis of $\mathrm{CH}$ selection process. Few of the $\mathrm{CH}$ selection algorithms are discussed in this section.

\section{LEACH}

Low Energy Adaptive Clustering Hierarchy (LEACH) is an energy-conserving routing protocol for WSN. It's one of the widely used dynamic clustering protocols. In $\mathrm{LEACH}$, sensor nodes form clusters and the $\mathrm{CH}$ act as routers to the sink. This is used to save energy since the transmission will only be done by $\mathrm{CHs}$ rather than all sensor nodes. In the LEACH protocol, selection of $\mathrm{CH}$ is done two phases [1][5]. 1. The setup phase with each node generates a random number between 0 and 1 . If the random number is smaller than the threshold value then that node become $\mathrm{CH}$. The threshold value is calculated based on the following equation:

$$
\mathrm{T}(\mathrm{n})=\left\{\begin{array}{lr}
\frac{p}{1-p *\left(r \bmod \frac{1}{p}\right)} & \text { if } n \in G \\
0 & \text { Otherwise }
\end{array}\right.
$$

$\mathrm{p}$ is the percentage of CHs and $\mathrm{r}$ is the current round in the $\mathrm{CHs}, \mathrm{G}$ is the group of nodes that has not been the $\mathrm{CHs}$ in the previous round $\mathrm{CH}$ and it will be the selected in the next rounds until all other nodes in the network becomes CHs. 2. In the steady phase, nodes send their data to the $\mathrm{CH}$ using a Time Division Multiple Access (TDMA) schedule. TDMA 
schedule allots time slots to every node. The $\mathrm{CH}$ need for aggregates the data and sends it to the BS.

LEACH achieves more reduction in energy dissipation compared to direct communication and four to eight times as compared to the minimum transmission energy routing protocol. LEACH employs dynamic clustering, which increases the lifetime of the system.

\section{PEGASIS}

Power-Efficient Gathering in Sensor Information Systems (PEGASIS) is a near optimal chain-based protocol. It is used to increase the network lifetime of each node by using collaborative techniques. This allows only local coordination between nodes and the bandwidth consumed in communication is reduced [11]. In PEGASIS, each node communicates only with a neighbor and takes turns transmitting to the BS [7]. In sensor networks, is used to reduce the amount of data transmitted between sensor nodes and the BS. Data synthesis combines one or more data packets from different sensor measurements to produce a single packet [8]. The idea in PEGASIS is to form a chain among the sensor nodes so that each node will receive from and transmit to a close neighbor. Gathered data moves from node to node, get fused, and eventually a designated node transmits to the BS [5].

Chain construction- The chain start from the BS, and then greedy approach is used to construct the chain.

Gathering data- Each round is selected randomly. If $\mathrm{N}$ is the number of nodes I mod $\mathrm{N}$ node is selected as a head node for round. Randomly selecting head node, it provides benefit as it is more likely for nodes to die at random locations thus providing robust network. When a node dies, chain is reconstructed to the dead node. Head node receives all the fused data and sends to the BS.

\section{TEEN}

This protocol organizes the sensor nodes into multiple levels of hierarchy. All sensor nodes are aggregated and transmitted by the $\mathrm{CHs}$ until it reaches the $\mathrm{BS}$, CHs are chosen depends on their remaining energy [13]. Here two data threholds are considered in order to minimize the number of transmissions and to increase the network lifetime. There are two thresholds used for these protocols.

1. Hard Threshold (HT)

2. Soft Threshold (ST)

HT is a threshold value for the sensed attribute. It is the absolute value of the attribute beyond which, the node sense the value must switch on its transmissions and report to its $\mathrm{CH}$. ST is a value of the sensed attribute which triggers the node. This node switch on its transmitter and to transmit.

The environment senses the nodes, the first time a parameter from the attribute set reaches its HT value. The node switches on its transmitter and sends the sensed data. The sensed value is stored in an internal node, called the sensed value (SV). The nodes will next transmit data in the current cluster level, only when both the following conditions are true:

1. The current value of the sensed attribute is greater than the HT.

2. The current value of the sensed attribute differs from SV by an amount equal to or greater than the ST.

Whenever a node transmits data, SV is set equal to the current value of the second attribute.

Thus, the HT tries to reduced the number of transmissions by allowing the nodes to transmit only when the sensed attribute is in the range of interest. The ST further reduces the number of transmission by eliminating all the transmissions. When there is little or no change in the sensed attribute once the hard threshold, which might have other-wise occurred.

\section{APTEEN}

Adaptive Periodic Threshold-Sensitive Energy Efficiency Sensor Network Protocol (APTEEN) is one of the protocol to select the $\mathrm{CH}$. In each cluster period, the cluster head first broadcasts the following parameters[9]:

Attributes: This is a set of physical parameters which the user is interested in obtaining data about the routing protocol.

Threshold: This parameter consists of a HT and a ST. HT is a particular value of an attribute beyond which a node can be triggered to transmit data or file. ST is a small change in the value of an attribute which can trigger a node to transmit data again.

Schedule: This is a TDMA schedule similar to the one used in, assigning a slot to each node.

Count Time: It is the maximum time period between two successive reports sent by a node. It can be a multiple of the TDMA schedule length and it accounts for the proactive component.

\section{HEED}

Hybrid Energy Efficient and Distributed (HEED) is a distributed cluster protocol. An important feature of HEED protocol is it to exploit the availability of multiple transmission power levels at sensor nodes. The HEED terminates in a constant number of iterations that is independent of networks diameter [10][13]. It only assumes the sensor nodes can control their transmission power level and not consider the distribution of nodes or about node capabilities. There are four essential objectives as follows: (1) increasing network lifespan, (2) steady number of iterations, (3) reducing control overhead, (4) generating well-distributed $\mathrm{CHs}$ and compact clusters. HEED choose a CHs based on a hybrid of two clustering parameters. The primary parameter is used to choose an initial set of CHs while the secondary parameter is used for breaking ties CHs.

\section{CONCLUSION}

Clustering is a technique, which reduces the energy consumption and provides stability in WSNs. The recent energy efficient clustering protocols designed for sensor networks are based on residual energy, average energy, location, density etc. The surveyed energy efficient clustering protocol is based on $\mathrm{CH}$ selection techniques. This has surveyed the state-of-art of different clustering algorithms in WSNs. Some energy efficient algorithms increase the network lifetime. There are wide range of challenges in wireless sensor networks.

\section{REFERENCES}

[1] Vishal Singh, "A Survey of Energy Efficient Clustering Algorithms in Wireless Sensor Networks," International Journal Of Engineering And Computer Science, Vol.5, pg. 17961-17966, 2016.

[2] M.Sheik Dawood et al, "A Survey On Energy Efficient Clustering Protocols for Wireless Sensor Networks," 
International Journal of Computer Science and Mobile Computing, Vol.3, pg. 1158-1163, 2015.

[3] Vinay Kumar, Sanjeey Jain and Sudarshan Tiwari, IEEE Member," Energy Efficient Clustering Algorithms in Wireless Sensor Networks:Survey," IJCSI International Journal of Computer Science, Vol. 8, No 2, pg. 259-268. Sep 2011.

[4] Firoj Ahamad, Rakesh Kumar," Energy Efficient Routing Protocols for Wireless Sensor Networks:A Review,” International Journal of Innovations \& Advancement in Computer Science, Vol. 4, pg. 165-171, Sep 2015.

[5] Swati Shamkumar, Prof. Vimal Shukla," A Review on Energy Efficient Routing Protocols in Wireless Sensor Networks," International Journal of Emerging Technology and Advanced Engineering, Vol. 4, ISSN 2250-2459, Pg. 653-657, March 2014..

[6] Sissy Annamma Johnson, Josmy George," A Survey on Different Types of Clustering Based Routing Protocols in Wireless Sensor Networks," Journal of Research, Vol. 2, ISSN 2395-7549, pg. 13-16, Nov 2016.

[7] Santal Pal Singh, S.C. Sharma,” A Survey on Cluster Based Routing Protocols in Wireless Sensor Networks," International Confrence in Advanced Computing Technologies and Applications, pg. 687-695, 2015.
[8] Sanjeev Kumar Gupta, Neeraj Jain, Poonam Sinha,” Clustering Protocols in Wireless Sensor Networks: A Survey," International Journal of Applied Information, Vol. 5, No-2, ISSN: 2249-0868, pg. 41-50, Jan 2013.

[9] Xu-Xun Liu," A Survey on Clustering Routing Protocols in Wireless Sensor Networks," School of Electronic and Information Engineering, ISSN 1424-8220, Aug 2012.

[10] Kunkunuru Udayakumar et al," Analysis of Various Clustering Algorithms in Wireless Sensor Networks," International Journal of Computer Science and Information Technologies, Vol. 6(2), pg. 1685-1691, 2015.

[11] Vandna Sharma, Payal Jain,” Various Hierarchical Routing Protocols in Wireless Sensor Network: A Survey,” IJCSMC, Vol. 2, Issue.5, pg. 63-72, May 2013.

[12] E.Prabashini, D.Sivakumar,” Energy Efficient Cluster Based Routing in Wireless Sensor Networks,” International Journal of Computer Science and Network Security, Vol. 16, No 3, pg. 125-128, March 2016.

[13] Punret Gurbani, Hansa Acharya, Prof. Anurag Jain,” Hierarchical Cluster Based Energy Efficient Routing Protocol for Wireless Sensor Networks: A Survey,” International Journal of Computer Science and Information Technologies, Vol.7(2), 2016. 